\title{
Utilisation de la technique éclair dans la thérapie EMDR : quatre exemples de cas
}

\author{
Philip Manfield \\ Université John F. Kennedy, Berkeley, Californie, États-Unis \\ Joan Lovett \\ Healthy Perspectives, Berkeley, Californie, États-Unis \\ Lewis Engel \\ San Francisco, Californie, États-Unis \\ David Manfield \\ EMDR Associates, Lake Oswego, Oregon, États-Unis
}

\begin{abstract}
Cet article présente la technique éclair, une nouvelle technique utilisée pendant la phase de préparation de la thérapie EMDR (désensibilisation et retraitement par les mouvements oculaires) pour faciliter le traitement de souvenirs traumatiques intenses auxquels les patients hésiteraient autrement à accéder. Les premières données, demeurant à confirmer, suggèrent que cette technique pourrait permettre aux patients d'accéder à ces souvenirs d'abord de manière la moins perturbante possible, réduisant leur intensité émotionnelle, afin de pouvoir ensuite les aborder plus pleinement et les traiter à l'aide de la thérapie EMDR. La technique semble aisément tolérée par les patients de tous âges, y compris les enfants ; elle paraît rapide et relativement indolore pour les patients, même ceux qui possèdent des souvenirs cibles particulièrement perturbants; elle s'enseigne facilement aux cliniciens. Elle se distingue par le fait que les patients qui évitent un souvenir terriblement perturbant peuvent se voir offrir une façon de le traiter sans devoir le ramener clairement à l'esprit. Quatre exemples de cas, dans lesquels quatre cliniciens différents ont utilisé la technique, sont présentés brièvement. Des suggestions sont formulées pour des études à venir. Cet article présente des hypothèses pour expliquer divers mécanismes d'action et évoque les effets en termes de la théorie de reconsolidation mnésique.
\end{abstract}

Mots-clés : technique éclair ; EMDR (désensibilisation et retraitement par les mouvements oculaires) ; reconsolidation mnésique ; subliminal ; mémoire de travail ; trauma

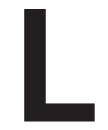

'EMDR (désensibilisation et retraitement par les mouvements oculaires) est une psychothérapie dévelopée et présentée par Francine Shapiro (1989), initialement pour la prise en charge de patients qui ont vécu des traumatismes isolés provoquant un état de stress post-traumatique (ESPT). La psychothérapie EMDR (Shapiro, 2001) est maintenant reconnue comme une méthode efficace permettant de traiter toute une gamme d'affections psychologiques liées au psychotrauma. Pendant la thérapie EMDR, le patient se centre sur l'incident perturbant tout en réalisant une tâche d'attention double dans l'instant présent, le plus généralement des mouvements oculaires $(\mathrm{MO})$ rapides guidés par les stimulations bilatérales (SBL) consistant à suivre des yeux les déplacements du doigt du thérapeute. Le protocole EMDR comprend huit phases : histoire du patient, préparation, évaluation, désensibilisation, installation, scanner corporel, clôture et réévaluation.

\section{Les huit phases de l'EMDR}

Les quatre premières phases

Cet article s'applique aux quatre premières phases de l'EMDR. Les deux premières, le recueil de l'histoire

This article originally appeared as Manfield, P., Lovett, J., Engel, L., \& Manfield, D. (2017). Use of the Flash Technique in EMDR: Four Case Examples. Journal of EMDR Practice and Research, 11(4), 195-205. Translated by Jenny Ann Rydberg. 
du patient et la préparation, permettent au thérapeute de formuler une conceptualisation du cas, de préparer le patient à la prise en charge EMDR et de sélectionner un souvenir cible approprié (cible) à traiter. La troisième phase, l'évaluation, est censée déterminer les composants du souvenir et établir des mesures de référence grâce auxquelles la progression du traitement pourra être estimée. L'échelle des unités subjectives de perturbation (SUD [subjective units of disturbance] ; Wolpe, 1958) permet d'évaluer le niveau de perturbation du patient. Pendant la quatrième phase, la désensibilisation, le souvenir est traité à l'aide de SBL et, parfois, de commentaires de cadence (P. Manfield, 2013 ; décrits dans Shapiro, 2001, p. 175), des commentaires brefs, à intervalles réguliers, sans signification particulière, tels que "Remarquez simplement », "Voilà » et «Bien».

\section{Les quatre dernières phases}

Lorsque le traitement EMDR se réalise avec succès, les trois phases suivantes se produisent après la désensibilisation complète d'un souvenir. L'installation renforce le point de vue adulte adaptatif qui est censé avoir remplacé la distorsion cognitive initialement associée au souvenir. Le scanner corporel vérifie l'existence de sensations perturbantes résiduelles liées au souvenir. La clôture prépare le patient à quitter la séance dans un état d'équilibre émotionnel et apporte des instructions pour le laps de temps entre la séance actuelle et la suivante. La huitième phase, la réévaluation, a lieu lors de la séance suivante, lorsque le thérapeute vérifie si les résultats du traitement ont perduré et si un traitement supplémentaire est nécessaire pour ce souvenir-là.

\section{Réparer le trauma intense}

\section{Traiter le trauma accablant}

Certains patients ont un matériel traumatique si perturbant qu'il leur est impossible de demeurer émotionnellement stable en y accédant et qu'ils tendent à devenir soit submergés soit inertes pendant le traitement. Plusieurs psychothérapies comprennent des éléments qui permettent aux patients de traiter le trauma accablant avec un niveau d'activation plus modéré. Certaines, parmi lesquelles on compte les SBL en EMDR, la méthode de comptage (Ochberg, 1996) et le comptage progressif (Greenwald, 2008), paraissent créer une tâche qui sollicite la mémoire de travail (MT) de sorte que les patients soient incapables de réaliser la tâche en même temps qu'ils maintiennent un souvenir vif et perturbant du trauma (Maxfield, Melnyk \& Hayman, 2008 ; Shapiro, 2001).
Dans la thérapie EMDR, diverses techniques ont été développées pour aider les patients dont l'implication dans leur prise en charge est mise à mal par une sur- ou sous-modulation. En plus d'une préparation approfondie, on applique certaines procédures lorsque la désensibilisation se trouve bloquée. Certaines techniques impliquent une exposition graduée ou la substitution du souvenir initial par une souvenir modifié moins perturbant (Forgash, 2004 ; Gomez, 2014 ; Shapiro, 2001). Certaines restreignent le temps pendant lequel les patients pensent au souvenir à une durée aussi courte que deux secondes, puis augmentent graduellement ce laps de temps (Greenwald, 2008 ; Kinowski, 2003 ; Knipe, 2008). D’autres renforcent les connexions de leurs patients à leur identité adulte positive pendant le retraitement pour que le matériel traumatique puisse être observé avec un certain recul plutôt que revécu (Forgash, 2004 ; Korn \& Leeds, 2002 ; U. Lanius, 2005 ; Leutner \& Cronauer, 2010 ; P. Manfield, 2010 ; P. Manfield \& Shapiro, 2003 ; Shapiro, 2001). Sont pertinentes dans cet article, les techniques de titrage jumelé (Kinowski, 2003) et la position de l'observateur (Shapiro, 2001).

\section{Titrage jumelé}

Dans son processus de titrage jumelé, Kinowski (2003) a adapté la technique d'oscillation du somatic experiencing de Levine (1997) à l'EMDR. Elle aide d'abord le patient à développer une connexion à une image ressource qui l'aide à se sentir plus résilient, puis le convie à restreindre son exposition au matériel traumatique en lui disant de n'aller que jusqu'à la lisière du trauma. De plus, elle limite la durée pendant laquelle le patient est exposé au souvenir, en guidant verbalement le patient en temps réel à travers les étapes consistant à se connecter à la ressource, à aller jusqu'à la lisière du trauma, puis à revenir à la ressource. Dans son protocole, les patients alternent de manière répétée entre la ressource et le matériel traumatique. Avec le temps, Kinowski rend les durées d'exposition de plus en plus longues et les focalise de plus en plus sur les éléments centraux du trauma. Tout au long de ce processus, elle évalue si chaque contact avec le trauma a été trop intense selon la difficulté rapportée par le patient à revenir vers l'état ressourcé.

\section{Maintenir la position de l'observateur}

Shapiro (2001) a déclaré que "les composants [de l'EMDR] ... sont conçus de sorte à convaincre la patiente qu'elle est plus que sa pathologie et qu'elle peut véritablement demeurer observatrice de ses effets jusque-là envahissants " (p. 141). Le rôle de 
l'observateur (orientation présente) semble prévenir la reviviscence de l'expérience traumatique douloureuse. Shapiro postule que la position de l'observateur améliore l'attention en pleine conscience.

\section{Recherches associées}

\section{Recherche sur la reconsolidation mnésique}

La reconsolidation mnésique est un terme permettant de décrire la manière dont les souvenirs ramenés en mémoire de travail depuis la mémoire à long terme sont de nouveau conservés en mémoire à long terme. Il s'agit d'un processus important car il peut expliquer comment des souvenirs altérés peuvent remplacer les originaux de manière permanente, permettant à des souvenirs perturbants de devenir bénins. Cette transformation permanente est désignée par le terme assez confus d'effacement (Elsey \& Kindt, 2017). En réalité, seule la nature traumatique du souvenir est " effacée»; le récit de l'événement demeure intact.

La recherche sur la consolidation mnésique apporte une certaine compréhension des conditions nécessaires à la survenue d'un effacement. Les études suggèrent que l'effacement de réponses conditionnées de peur chez diverses espèces animales ne se produit que lorsque l'animal accède au souvenir original qui a provoqué la réponse de peur et qu'il existe aussi une erreur prédictive (Dudai, 2004 ; Pedreira, PérezCuesta \& Maldonado, 2004). En d'autres termes, quand l'animal rencontre un stimulus similaire à celui qui était présent dans le souvenir original émotionnellement associé, il extrait le souvenir original de la mémoire à long terme vers la mémoire de travail. Ensuite, pour que l'effacement ait lieu, l'animal doit rencontrer une information nouvelle qui, on le suppose, nécessite une actualisation du souvenir initial pour le rendre plus juste. Les chercheurs ont identifié une période allant jusqu'à six heures pendant laquelle le souvenir en mémoire de travail devient labile et l'actualisation est susceptible de se produire (Elsey $\&$ Kindt, 2017).

Ecker, Ticic et Hulley (2012) ; Hupbach, Gomez, Hardt et Nadel (2007); Lee (2009); Sevenster, Beckers et Kindt (2013) et d'autres relatent des données qui laissent penser que l'effacement de souvenirs chez les humains requiert également à la fois une extraction et une erreur prédictive. L’erreur prédictive (p. ex. «Je suis en sécurité maintenant » contredit la prédiction initiale «Je serai blessé(e) ») est incorporée dans le souvenir qui a été extrait et le souvenir altéré peut alors remplacer le souvenir original quand il est de nouveau sauvegardé (reconsolidé) en mémoire à long terme.
La théorie de la reconsolidation mnésique suggère que cette altération du souvenir original, une fois reconsolidée, est permanente.

\section{L'EMDR peut altérer définitivement des souvenirs traumatiques}

L'impact observable de la thérapie EMDR quand elle est efficace suggère qu'elle produit un changement permanent (effacement) et qu'elle répond donc à chacune de ces conditions requises. Elle implique le plein accès au souvenir traumatique pendant les phases d'évaluation et de désensibilisation de la prise en charge en EMDR et elle implique une expérience pour le patient qui contredit ses attentes. En EMDR, l'expérience de se rappeler le souvenir avec des SBL simultanées ainsi que l'absence de reviviscence de l'affect envahissant ou du sentiment intense de danger ou de honte constituent probablement l'erreur prédictive requise.

\section{Caractéristiques nécessaires de l'extraction (remémoration) de souvenirs}

La recherche sur la reconsolidation mnésique a montré que le souvenir doit être extrait (remémoré) pour que l'effacement ait lieu, mais elle demeure vague pour définir ce en quoi consiste cette extraction nécessaire. Dans la thérapie EMDR, par exemple, l'extraction ou remémoration de souvenirs est explicite, manifeste et le plus souvent émotionnelle.

La phase d'évaluation encourage l'extraction (remémoration) de souvenirs lorsque le patient évoque les aspects visuels, cognitifs, émotionnels et somatiques du souvenir cible qui, ensemble, avec les SBL, contribuent à permettre au patient de retrouver le souvenir. En effet, la plupart des cliniciens EMDR expérimentés attesteront que quand les patients évitent d'accéder pleinement à un souvenir cible pendant la thérapie EMDR, le progrès est souvent freiné.

Est-il toutefois nécessaire que l'extraction de souvenirs comprenne une telle connexion consciente et émotionnelle aux souvenirs ? Au moins une étude animale conclut que la reconsolidation et l'effacement peuvent s'accomplir même si le rappel affectif («l'expression ») du souvenir traumatique est bloqué chimiquement (Barreiro, Suárez, Lynch, Molina \& Delorenzi, 2013). Ceci semble également être le cas avec la technique éclair (TE) décrite ci-dessous.

Quelle est la durée minimale pendant laquelle un souvenir doit être tenu en mémoire de travail pour satisfaire le critère d'extraction ? Un large corpus de recherches démontre que 4 millisecondes seulement, 
quatre millièmes de seconde, d'accès à un stimulus visuel suffisent à stimuler une pleine extraction d'informations et un traitement du stimulus (D. Manfield, 1986 ; Silverman \& Weinberger, 1985 ; cf. Mansfield, 1997, pour une discussion approfondie des recherches pertinentes). De plus, un stimulus visuel de moins de 37 millisecondes a peu de chances d'être perçu consciemment ou compris ; néanmoins, il aura un impact psychologique manifestement plus important qu'une stimulation qui est consciemment reconnue et qui en réalité n'exerce pas d'impact significatif (Mansfield, 1997). La plupart de ces recherches ont été effectuées avec des messages verbaux visuels subliminaux, tant positifs que négatifs, mais certaines impliquaient une exposition subliminale à des stimuli constitués d'images visuelles (Meyer \& Waller, 1999). Bien que toutes les recherches sur les messages subliminaux se centrent sur des stimuli extérieurs perçus, il est raisonnable de soupçonner que des seuils temporels similaires s'appliquent aux stimuli qui trouvent leur origine dans la mémoire à long terme.

Dans ces recherches, ce qui importe à cet article est que (a) au moins chez les animaux, il est possible que des souvenirs traumatiques puissent être extraits sans leur composant émotionnel et être malgré tout altérés et reconsolidés ; (b) il est possible d'accéder à une image ou à un message de manière suffisamment complète pour qu'ils aient un impact significatif sur le spectateur, même si l'exposition était si brève que le spectateur ne se souvient pas de ce qu'il a vu; et (c) les images ou les messages qui apparaissent assez longtemps pour être reconnus consciemment n'ont en réalité aucun effet observable sur le spectateur. À partir des résultats de ces recherches et de notre expérience avec la TE, nous pensons que l'exposition extrêmement brève au souvenir traumatique pendant la TE peut suffire à répondre au critère d'extraction proposé par Ecker et coll. (2012), Lee (2009) et d'autres.

\section{La technique éclair}

La technique éclair est employée pendant la phase de préparation de l'EMDR en tant que moyen rapide et relativement indolore de réduire l'intensité de souvenirs extrêmement perturbants afin qu'ils puissent être aisément traités lors des phases subséquentes de la prise en charge EMDR standard. Elle est recommandée pour les patients qui appréhendent fortement d'accéder à leur matériel traumatique, qui se dissocient lorsqu'ils y accèdent, qui sont submergés par les émotions ou qui résistent à l'idée de revisiter leurs souvenirs. On peut aussi l'utiliser chez certains patients au lieu d'activités de préparation plus longues. Le but de l'intervention consiste à réduire, en faisant souffrir le moins possible, la perturbation associée à un souvenir cible jusqu'à un niveau auquel le patient n'est plus résistant à l'idée d'y accéder pleinement et de le traiter en EMDR standard. Les patients sont informés qu'il peut s'agir d'une façon de réduire la détresse liée au souvenir sans devoir y penser. $S$ 'ils répondent avec un scepticisme marqué, le thérapeute indique que la procédure pourrait ne pas fonctionner mais qu'ils n'ont rien à perdre - sauf une dizaine de minutes. L'intervention peut durer entre dix et 45 minutes (Tableau 1).

Avant qu'un souvenir ne devienne considérablement activé, le patient est invité à démarrer à partir d'un état ressourcé ou relativement neutre et, quand il est prêt, à revisiter son souvenir en un éclair, en une petite fraction de seconde, puis d'informer le thérapeute quand il est revenu à l'état neutre ou ressourcé. L'état ressourcé peut être généré par toute pensée, souvenir ou image qui apporte au patient un sentiment de bienêtre ou de calme. Avant et pendant l'éclair, des SBL sont employées pour guider le patient dans la réalisation de mouvements oculaires lents (c.-à-d. environ deux à trois secondes pour chaque passage gauche-droite-gauche). On demande au patient d'indiquer quand il est revenu à l'état neutre ou ressourcé. La série complète consiste généralement en quatre à cinq va-et-vient, le patient accédant au souvenir en un éclair après le troisième ou quatrième va-et-vient ; toutefois, certains patients peuvent avoir besoin de plus de temps au départ.

\section{L'éclair doit rester bref}

On avertit le patient de ne pas penser au souvenir ou de s'y exercer avant l'éclair, mais plutôt, quand il est prêt, de simplement penser au souvenir ou à la perturbation pendant une toute petite fraction de seconde puis de revenir à l'état neutre ou ressource. L'éclair peut être décrit comme une étincelle ou un scintillement. On peut dire aux patients que l'erreur la plus fréquente au niveau de l'éclair consiste à accéder trop longtemps au souvenir car cela peut rendre l'éclair plus perturbant que voulu. On peut les rassurer en disant que ce n'est pas grave s'ils laissent passer l'éclair si vite qu'ils ne sont pas sûrs de s'être réellement connectés au souvenir et que c'est même souvent dans ces cas-là que l'effet le plus rapide est obtenu. Une métaphore qui fonctionne bien pour décrire l'éclair est celle du passage d'un doigt à travers la flamme d'une bougie. Si on le fait rapidement, cela ne devrait pas susciter de douleur.

\section{Stimuli subliminaux}

Il est possible que quand un patient est convié à accéder à un souvenir si rapidement qu'il n'apparaît que 
de manière floue et fugace, l'effet soit similaire à celui identifié dans la recherche sur les messages subliminaux qui vient d'être mentionnée (Mansfield, 1997). Cette recherche a montré que si le stimulus demeurait visible assez longtemps pour que le patient le reconnaisse consciemment, alors aucun effet psychologique n'était observé. Pour avoir un effet, le message doit être assez bref pour que le sujet ne puisse pas y penser consciemment et s'en défendre. De manière comparable, la TE semble fonctionner le mieux quand l'éclair est si rapide que le patient n'a pas le temps de réfléchir au souvenir ou de se le rappeler clairement.

Comme dans le jumelage titré, on demande aux patients s'ils ont eu du mal à revenir à l'état neutre après chaque éclair. Si tel est le cas, on leur demande de rendre l'éclair suivant beaucoup plus bref. Après une série, le thérapeute ne demande pas le niveau du SUD ni "Qu'est-ce qui est là maintenant ? " car ces questions peuvent encourager les patients à penser à la perturbation, ce qui va à l'encontre de l'intention de la TE. Quand les patients peuvent revisiter le souvenir en un éclair et revenir à l'état neutre sans difficulté à plusieurs reprises, le thérapeute peut les convier à faire trois éclairs par série. Après environ trois de ces triples éclairs, on demande aux patients s'ils remarquent une différence au niveau de la perturbation suscitée par le souvenir cible. Si les patients signalent une perturbation considérablement affaiblie ou une difficulté à accéder au souvenir, le thérapeute peut demander quel est le niveau de SUD associé au souvenir. Quand la perturbation initiale a été réduite, le passage à la phase d'évaluation de l'EMDR permet aux patients de traiter la perturbation qui demeure, dont une partie peut être associée à des canaux secondaires du souvenir cible et une autre partie à des distorsions cognitives ou à des sensations corporelles liées au souvenir cible.

\section{Les abréactions sont rares}

Généralement les patients ne deviennent pas activés pendant le traitement TE. À de rares occasions au cours d'un éclair initial, un patient se connecte au souvenir plus longtemps que ce qui lui a été demandé et commence à abréagir. À ce jour, de telles abréactions ont été légères parce que ces patients n'accédaient pas encore à la puissance entière du souvenir et l'intensité émotionnelle s'est dissipée au cours d'une ou deux séries complètes de MO rapides avec des commentaires de cadence. Entre autres, les MO rapides avec des commentaires de cadence semblent aider le patient à contenir les affects à mesure qu'ils émergent, comme ils semblent le faire pendant la phase 4 EMDR.

\section{La technique éclair démarre à partir d'un état neutre ou ressourcé}

Idéalement, la TE démarre à partir d'un état neutre ou ressourcé au cours de la phase de préparation de la thérapie EMDR. Au départ, pour les souvenirs particulièrement perturbants, le thérapeute peut aider le patient à créer un lieu sûr ou calme sur lequel se centrer avant la TE. Il invite spécifiquement le patient à ne pas décrire ni même penser au souvenir cible avant l'éclair et à ne pas s'entraîner ou réfléchir à ce à quoi il pensera pendant l'éclair. Ceci sert à empêcher la stimulation de la peur d'un affect envahissant chez le patient, car dès que cette peur est devenue forte chez ces patients, la probabilité d'évitement ou de dissociation augmente.

Si le patient est un peu activé au départ, on peut l'inviter à penser à l'état sûr, calme ou agréable, puis permettre aux MO lents (Kreyer \& Egon, 2008 ; Schubert, Lee $\&$ Drummond, 2011) de l'aider à se détendre davantage avant de tenter de réaliser un éclair. La plupart des patients semblent capables d'y arriver.

\section{Vitesse des mouvements oculaires}

Au cours de la TE, les MO sont lents, environ 2 à 3 secondes par va-et-vient. Cette vitesse est trop lente pour solliciter la mémoire de travail (Van Veen et coll., 2015), donc elle ne semble pas empêcher l'accès bref au souvenir traumatique qu'effectue le patient pendant la TE. Comme nous l'expliquerons plus loin, il

\section{TABLEAU 1. SOMMAIRE DU TRAITEMENT DE QUATRE ÉVÉNEMENTS TRAUMATIQUES}

\begin{tabular}{lllll}
\hline Cas & SUD pré-TE & SUD post-TE & ESPT & Minutes de TE + EMDR \\
\hline 1 & 10 & 0 & Oui & 20,45 \\
2 & 10 & 0 & Oui & 10 \\
3 & 9 & 0 & Oui & 45 \\
4 & 10 & 0 & Oui & 45 \\
\hline
\end{tabular}

Note. ESPT = état de stress post-traumatique ; Minutes de TE + EMDR = temps consacré au traitement d'un souvenir donné $;$ SUD = unités subjectives de perturbation ; $T E=$ technique éclair. 
n’est pas souhaitable de solliciter la mémoire de travail avec des MO plus rapides dans la TE. Les MO lents semblent en effet aider le patient à se détendre dans un état neutre ou ressourcé avant d'effectuer l'éclair (Kreyer \& Egon, 2008), ce qui est utile pour la TE, et cela semble apporter une structure utile à de nombreux patients.

Les patients sont rassurés par la description de la technique éclair

Afin d'aider les patients à comprendre que la TE est conçue pour les aider à ne pas devenir submergés, les thérapeutes peuvent leur dire que leur contact avec la perturbation sera si bref qu'ils n'auront pas le temps (a) de se sentir mal, (b) de faire venir une image bien définie, ou (c) d'avoir des pensées désagréables. Il n'y aura pas assez de temps pour que le souvenir devienne intrusif. Avec ces paroles rassurantes, les patients sont généralement prêts à accéder aux souvenirs qu'ils auraient peut-être évité autrement. Après 6 à 12 éclairs adéquatement brefs, les patients signalent généralement une diminution significative de la perturbation associée au souvenir et ils sont prêts à passer à la phase d'évaluation de l'EMDR et au reste du protocole standard.

\section{Description de quatre cas}

Quatre cas, dans lesquels la TE était utilisée par quatre thérapeutes différents lors de la phase de préparation de la prise en charge EMDR, seront présentés brièvement. Les cibles dans les quatre cas avaient un niveau de SUD évalué à 9 ou 10. Dans chaque cas, la cible était extrêmement perturbante et le thérapeute anticipait que le traitement allait se montrer difficile, soit parce que les patients avaient tendance à « tout couper " plutôt qu'à traiter (cas 1 et 2), soit parce qu'ils se disaient réticents à penser à la cible (cas 3 et 4 ). Il était attendu que la TE réduise les défenses du patient et rende le traitement rapide, sans perturbation notable pour le patient. Dans tous les cas sauf le cas 2, où le patient était un enfant, le protocole EMDR standard a été utilisé après la réduction du SUD à un niveau modéré. Les résultats dans les quatre cas sont demeurés stables jusqu'au suivi.

\section{Description du cas 1}

Présentation du patient. Martin était un homme de 35 ans, intelligent, éduqué, qui a consulté un an après une collision arrière qui a endommagé sa colonne vertébrale et nécessité de nombreuses interventions chirurgicales. Bien que son état médical ne soit toujours pas résolu au moment de sa consultation en psychothérapie, il pensait que s'il obtenait une prise en charge pour l'aider à faire face au trauma de l'accident, cela pourrait rendre sa vie présente moins perturbante. Il a décrit sa vie comme un désastre, disant qu'il dormait mal et mangeait peu, avait des flash-backs dès qu'il montait dans une voiture et qu'il n'avait pas pu garder de poste à plein temps depuis l'accident.

Traits dissociatifs. Martin indiquait qu'il avait été moyennement dissociatif depuis un jeune âge, devenant par moments désorienté et ne sachant pas où il était ni pourquoi. Il rapportait également qu'à certains moments, il était incapable de ressentir la douleur physique. Le diagnostic précis de ses symptômes dissociatifs n'était pas clair. Il répondait aux critères diagnostiques de l'ESPT. Il évaluait l'accident à 12 sur l'échelle du SUD de 0 à 10. Néanmoins, il semblait posséder bien des ressources, ce qui se manifestait par sa capacité à évoquer à une gamme très riche de souvenirs positifs.

Les signes dissociatifs d'ESPT de Martin sont apparus bien avant l'accident. Cependant, Martin indiquait qu'il se sentait assez présent dans la pièce de consultation tant que rien ne le faisait penser à l'accident. Les moindres éléments rappelant l'accident provoquaient toutefois souvent la reviviscence de l'accident et un envahissement. La partie la plus perturbante de l'accident pour lui était le moment de l'impact.

Décision d'employer la technique éclair. Martin aurait certainement tiré profit d'une prise en charge continue comprenant un renforcement des ressources, de la psychoéducation et un travail avec les parties dissociatives. Il ne se voyait cependant pas s'impliquer dans un effort psychothérapeutique à long terme. Le thérapeute (PM) souhaitait apporter le soulagement immédiat que Martin demandait spécifiquement.

Le thérapeute avait anticipé que le passage à la phase d'évaluation de la thérapie EMDR impliquerait le fait de penser à l'accident et amènerait probablement Martin à le revivre puis à se fermer, ce qui correspondait à sa manière habituelle de se protéger. Plutôt que de s'engager dans un processus élaboré de gestion de la dissociation, de développement des ressources du patient et de dosage de la perturbation associée au souvenir, il a été décidé d'employer la TE en préparation du protocole EMDR standard afin de tenter d'obtenir une diminution relative rapide $d u$ haut niveau de perturbation immédiate.

Vingt minutes de technique éclair. Aucune phase d'évaluation n'a été tentée avant d'employer la TE. Après quelques exercices de stabilisation, il restait vingt minutes dans la séance et la TE a été amorcée. Le patient était invité à se centrer sur la sensation 
d'être en sécurité et confortable, puis à revisiter en éclair, de manière extrêmement brève, le souvenir de l'impact de l'accident, et enfin à revenir à l'état confortable. Le thérapeute soulignait l'importance de rendre l'éclair si rapide qu'il n'y aurait pas assez de temps pour que le souvenir devienne perturbant. Il n'y aurait pas non plus assez de temps pour se rappeler des séquences d'action du souvenir ni même pour réfléchir au souvenir.

La première série éclair était la seule pour laquelle Martin a signalé une difficulté à revenir à l'état neutre, même s'il y est parvenu. Par la suite, il était systématiquement capable de revenir aisément à l'état calme. À la quatrième série, il signalait que le souvenir semblait lointain et qu'il avait du mal à y accéder. À la $14^{e}$ série, il avait du mal à retrouver dans sa mémoire l'expérience de l'impact de l'accident et il semblait qu'il était prêt à traiter le souvenir entier car son attention s'est déplacée vers l'expérience effrayante de voir l'autre voiture s'approcher dans son rétroviseur avant l’impact. La durée prévue de la séance s'était écoulée et il a été convenu que cet autre aspect du souvenir serait traité lors de la séance suivante. L'humeur de Martin est demeurée régulière pendant le traitement et s'est maintenue ainsi jusqu'à la fin de la séance. Avant de partir, il a indiqué se sentir stable avec un sentiment de bien-être.

Traitement EMDR subséquent sans technique éclair. Lors de la séance suivante, deux semaines plus tard, Martin a indiqué qu'il n'avait maintenant aucune réaction quand il pensait à l'accident qui était le sujet de ses flash-backs depuis un an. Il éprouvait très peu de difficulté en se déplaçant en voiture mais il était toujours déclenché par le fait de voir du mouvement dans le rétroviseur. Bien que cet aspect du souvenir de l'accident fût décrit avec un SUD de 9, il était évalué comme moins perturbant que l'avait été le souvenir de l'impact et le patient ne semblait pas résistant à l'idée de le traiter directement avec le protocole EMDR standard sans TE. À la fin de la séance, le SUD était décrit à 0 .

Technique éclair nécessaire. Lors de la réévaluation une semaine plus tard, cependant, le SUD associé à l'image dans le rétroviseur était évalué à 6 . Le thérapeute en a déduit que le SUD de 0 rapporté dans la séance précédente était le résultat d'une dissociation pour éviter l'intensité de la perturbation et que même le SUD décrit maintenant par Martin à 6 aurait été plus élevé s'il s'était autorisé à " entrer dedans ». La TE lui a de nouveau permis de tolérer la perturbation sans l'éviter. Avec la TE, la perturbation était réduite à un vrai 6 et le reste du protocole EMDR a ensuite permis de réduire le SUD à 0 . À la fin de la séance, le patient avait le sentiment que le souvenir était complètement résolu.

Suivi. La semaine suivante, Martin n'éprouvait toujours aucune difficulté à penser à un aspect quelconque de l'accident et le SUD était toujours évalué à 0 . Le thérapeute lui a rappelé que la première fois qu'ils avaient traité le souvenir du rétroviseur, le SUD était descendu à 0 , mais qu'il était remonté à 6 à la séance suivante. Il a demandé si Martin pensait qu'il était possible que cela se reproduise. Martin l'a remercié d'avoir posé la question et a ajouté : «Non, cette fois, c’est différent. Je ne sursaute pas dans la voiture ni rien ». Ces résultats se sont maintenus au suivi après trois mois.

\section{Description du cas 2}

Histoire $\boldsymbol{d} u$ patient. Mason était un garçon de six ans dont les parents adoptifs souhaitaient qu'il suive une thérapie centrée sur l'attachement et l'EMDR. Auparavant, Mason avait suivi une thérapie par le jeu pendant deux ans mais il avait toujours des problèmes de colères prolongées une ou deux fois par semaine, de comportements agressifs, d'accumulation de nourriture, d'hypervigilance, d'énurésie nocturne, d'une faible estime de soi, d'un sommeil perturbé et d'une peur intense du noir qui rendait le coucher très difficile. Ces symptômes étaient cohérents avec une histoire de trauma développemental complexe ainsi qu'un diagnostic d'ESPT et de trouble de l'attachement.

La peur intense du noir de Mason semblait provenir d'une expérience qui s'est déroulée dans l'une de ses familles d'accueil, lors de laquelle il était enfermé seul la nuit dans sa chambre dans le noir. Il se souvenait avoir été paniqué de se retrouver seul dans le noir et incapable de quitter sa chambre pour aller aux toilettes, alors cette nuit-là il a fait pipi au lit. Il était encore perturbé par ce souvenir, malgré son adoption par une famille sûre et aimante.

Niveau de perturbation. La thérapeute (JL) a demandé à Mason d'indiquer dans quelle mesure c'était troublant de se rappeler avoir été enfermé seul dans sa chambre dans le noir, utilisant pour l'évaluation du SUD la distance entre ses mains, les mains rassemblées signifiant calme et neutre, et les mains écartées de 25 $\mathrm{cm}$ reflétant la détresse maximale ( $\mathrm{SUD}=10)$. Mason a écarté ses bras autant qu'il le pouvait pour montrer sa détresse envahissante. Comme le SUD était si élevé et qu'il avait été tant traumatisé depuis un jeune âge, la thérapeute craignait que Mason se sente submergé si elle tentait de faire un traitement EMDR avec lui sans développement de ressources ou TE préalable.

Établissement de la sécurité présente. La thérapeute a commencé par construire des ressources et des 
mesures pour établir la sécurité présente. Elle a demandé au père de Mason si Mason serait un jour enfermé dans une pièce sombre dans leur maison et, bien sûr, le père l'a rassuré que cela ne se produirait jamais. Elle a ajouté : "Tu peux donc te détendre la nuit et savoir que tu es en sécurité ». La thérapeute a suggéré que le papa de Mason mette son bras autour de lui pour le rassurer qu'il se trouve en sécurité maintenant. Elle a demandé à Mason de sentir ses pieds sur le sol et le bras de son papa sur ses épaules, et a dit : «Ton papa et toi et moi sommes dans mon cabinet maintenant, et tu es en sécurité et tout va bien. Tu peux le ressentir?». Mason a fait oui de la tête.

Mise en auvre de la technique éclair. La thérapeute a demandé à Mason de " jeter un coup d'œil rapide » sur sa détresse d'avoir été enfermé dans une pièce sombre dans la famille d'accueil, puis de revenir rapidement au fait de se trouver dans son cabinet avec son papa et elle. Elle lui a indiqué de jeter un coup d'œil si rapide qu'il n'aurait pas le temps de ressentir ou de penser quoi que ce soit, puis de revenir et de dire : "Je suis de retour » ou «Éclair». Pendant qu’il « jetait un coup d'œil ", elle lui tapotait les genoux, et quand il disait " je suis de retour ", elle s'arrêtait. Puis elle lui demandait de sentir son papa le tenir et de sentir ses pieds sur le sol. Quand il faisait oui de la tête, elle poursuivait : " Es-tu prêt à jeter un coup d'œil ? ». Quand il indiquait que oui, la séquence était répétée, poursuivant l'alternance entre se sentir présent et en sécurité, et « jeter un coup d'œil ».

Résultats. En l'espace de dix minutes, le SUD de Mason est passé de 10 à 0 , et il disait que le souvenir ne le dérangeait plus. Un traitement extrêmement rapide n'est pas rare chez les enfants, mais cette séance se démarquait car, pendant les éclairs, Mason ne paraissait éprouver de perturbation apparente. Le reste du protocole EMDR standard a été omis car il ne semblait plus y avoir d'autres images, émotions ou sensations physiques perturbantes. Le SUD était de 0 et le scanner corporel était neutre. L'intention avait été de poursuivre avec le protocole EMDR complet pour la désensibilisation et le retraitement, mais cela n’a pas été nécessaire. Le fait de ne pas avoir accès aux émotions ni aux sensations physiques était un avantage clair dans le travail avec cet enfant qui avait des antécédents traumatiques si étendus.

Suivi. Lors du suivi après une, deux et trois semaines, les parents de Mason ont indiqué que le coucher se passait beaucoup plus facilement et que Mason n'avait plus peur du noir, ce qu'il a confirmé. Il dormait également une heure de plus par nuit, ce qui reflétait peut-être une détente accrue. Ces résultats se maintenaient après six mois.

\section{Description du cas 3}

Présentation de la patiente. Jenna était une femme de 34 ans, directrice des ressources humaines dans une petite entreprise technologique. Sa sœur aînée avait été sauvagement assassinée par un compagnon jaloux intoxiqué, trois ans auparavant. Jenna avait été dépressive, irritable et préoccupée à un degré tel que sa performance au travail en était affectée et que son mariage se trouvait en difficulté.

Prise en charge initiale. Après le recueil des antécédents, l'obtention de la liste de ses dix pires expériences, l'établissement d'une personne sûre et d'un conteneur, et après lui avoir enseigné quelques compétences de communication conjugale au cours des deux premières séances, lors de la troisième séance, le thérapeute (LE) a amorcé le traitement des souvenirs traumatiques. Un souvenir traumatique qui a démarré avec un SUD de 6 a pu être traité à l'aide du protocole EMDR standard.

Perturbation envahissante. Le deuxième souvenir, le meurtre de sa sœur, était d'abord évalué avec un SUD de 7 mais se trouvait manifestement à un niveau supérieur. Deux séances d'EMDR standard ont été nécessaires à la résolution complète. Toutefois, elle évitait de se centrer sur l'aspect le plus douloureux de cette série d'événements, l'identification du corps meurtri de sa sœur à la morgue municipale. Cette dernière partie de l'expérience était si terriblement douloureuse qu'elle en subissait quotidiennement des souvenirs intrusifs et elle disait qu'elle ne voulait tout simplement pas la faire venir à l'esprit, ce qui aurait été nécessaire pour l'EMDR standard. Elle répondait clairement aux critères de l'ESPT.

Traitement par la technique éclair. Comme elle a appris qu'elle pouvait utiliser la TE sans avoir besoin de se rappeler clairement le souvenir, elle a accepté de l'essayer. Elle évaluait son SUD à 9. Après les trois premières séries de $\mathrm{TE}$, elle ne rapportait aucun changement et décrivait une certaine difficulté à revenir des expositions éclair. Elle a été conviée à réduire le temps de contact avec le souvenir. À la cinquième série, elle était capable d'accéder au souvenir pendant l'éclair d'une fraction de seconde, sans voir de détail ni entendre de dialogue.

Les séries éclair suivantes étaient à peine perturbantes. Après la neuvième série éclair, le SUD était de 2. Après quatre séries supplémentaires, elle pouvait voir l'image sans éprouver de perturbation. "Cela a toujours l'air terrible, mais je n'ai juste pas la douleur ». D'autres canaux de la cible n'étaient toujours pas résolus, dont un avec un SUD évalué à 7, et les phases EMDR standard d'évaluation, de désensibilisation et 
d'installation ont permis d'achever le traitement. Le SUD était alors décrit à 0 et il ne semblait plus y avoir de perturbation du tout à la fin de la séance de $45 \mathrm{mi}$ nutes. Le scanner corporel était neutre.

Suivi. Lors du suivi après dix jours, la patiente indiquait que sa réactivité vis-à-vis du souvenir avait complètement disparu. Ces résultats étaient maintenus lors du suivi après six mois.

\section{Description du cas 4}

Présentation $d u$ patient. Charles était un homme de 48 ans qui s'est présenté avec une hypervigilance, des cauchemars et des conduites d'évitement. Il se décrivait également comme ayant été dépressif durant la plus grande partie de sa vie adulte. Le diagnostic principal était un ESPT provenant d'une fusillade au volant au début de la vingtaine. Pour des raisons devenues apparentes ultérieurement, le patient n'était pas prêt à décrire les détails de cet incident à son thérapeute (DM). Plus tard, il a divulgué, au cours du traitement, que l'un de ses pairs avait été tué par balle. Charles a été visé par des coups de feu automatiques et se rappelait avoir couru pour sauver sa vie. Depuis l'incident, il décrivait qu'il " restait aux aguets », « surveillait la pièce ", s'assurant de connaître les issues en tout temps.

Choix d'employer la technique éclair. Charles était d'accord pour dire que cet incident était sans aucun doute à l'origine de ses symptômes actuels, dont notamment ses flash-backs, son évitement et son hypervigilance. Néanmoins, il demeurait ambivalent à l'égard de l'EMDR et ne voulait pas du tout revisiter ce souvenir. Après avoir utilisé avec succès les procédures EMDR standard pour cibler du matériel moins menaçant lors de la deuxième séance, il a été proposé à Charles qu'une nouvelle approche de l'EMDR pourrait lui permettre de s'approcher du souvenir de la fusillade au volant, sans être submergé par les détails. Charles a accepté de participer à une troisième séance mais a avoué par la suite qu'il a failli ne pas s'y présenter.

Mise en ouvre de la technique éclair. Le patient n'a pas été invité à identifier le moment traumatique, ni à créer une image cible. Aucune phase d'évaluation n'a été réalisée. Dans une référence indirecte au souvenir, le thérapeute l'a simplement invité "à trois » à faire un éclair sur cela. De manière caractéristique de la TE, à mesure que le SUD diminuait, il n'y a eu aucune abréaction apparente ni de changement de l'émotion manifeste. En d'autres termes, il n'y avait aucune indication visible que quelque chose se passait. Au départ, le patient décrivait un SUD à 12 sur l'échelle de 0 à 10 . Après le premier éclair et la série de $\mathrm{MO}$ associée, il a décrit peu de changement. Cependant, la deuxième série a provoqué un commentaire sur le fait que le souvenir était légèrement moins perturbant, et plusieurs séries supplémentaires ont réduit la perturbation à 6 .

À ce moment-là, Charles était prêt à évoquer les détails de son souvenir et le traitement s'est poursuivi à l'aide des procédures EMDR standard sans TE. Les séries subséquentes se sont centrées sur les flaques de sang, le bruit des balles et la mort de son ami. Le traitement d'événements présents et futurs potentiels suscitant une perception irrationnelle de danger a permis de renforcer son sentiment de sécurité.

Suivi. La réévaluation la semaine suivante n'a identifié aucune perturbation associée à ce souvenir. Charles se sentait plus détendu, avec une résolution complète de sa peur et de son hyperactivation. Ces résultats étaient maintenus lors du suivi après 3 mois.

\section{Discussion}

\section{Sommaire}

Quatre exemples de cas ont été décrits, concernant des patients atteints d'ESPT provoqué par des événements extrêmement perturbants, Dans chaque cas, il était évident que le patient évitait consciemment ou inconsciemment d'accéder à la perturbation, rendant le recours à la TE idéal, car elle contourne la résistance du patient. Dans le cas 1, après avoir traité l'aspect le plus perturbant d'un accident sur la voie publique chez un patient modérément dissociatif, à l'aide de la TE, l'EMDR sans TE était employée pour traiter le deuxième aspect le plus perturbant de l'accident, et le traitement n'a pas abouti. Lors de la séance suivante, la TE a été utilisée avec succès sur cet aspect du souvenir, suivie de la procédure EMDR standard. Dans les quatre cas, la TE a permis de réduire rapidement les niveaux de perturbation associés des patients et les patients n'ont éprouvé que très peu de perturbation au cours du traitement par la TE (cf. tableau 1).

\section{Mécanismes d'action proposés}

Mécanismes proposés multiples. La plupart des patients atteints d'un état de stress post-traumatique revivent des aspects de leurs souvenirs traumatiques lorsqu'ils y pensent (Hackmann, Ehlers, Speckens \& Clark, 2004 ; R. A. Lanius et coll., 2010 ; Shapiro, 2001). Nous suggérons que l'une des raisons de l'efficacité de la TE est qu'elle empêche ces reviviscences des souvenirs traumatiques en réduisant considérablement le 
laps de temps disponible pour accéder au souvenir. De plus, la TE ne permet pas assez de temps au patient pour faire revenir un souvenir clair de la cible ou pour y penser. Nous pensons que cet aspect de la TE interrompt les défenses conscientes contre l'accès au souvenir. Aussi, l'alternance répétée entre l'état présent neutre et l'accès au souvenir passé avec assez peu d'émotion souligne que "cela, c'était alors, et ceci, c'est maintenant». Bien que nous ne puissions pas exclure la possibilité que les effets de la TE pourraient s'expliquer par la MT, à notre avis, la MT ne peut pas rendre compte des effets. Étant donné la rapidité des effets, nous pensons fermement que la TE possède un mécanisme d'action unique.

Ecker et coll. (2012) et Lee (2009) ont suggéré que pour une reconsolidation mnésique efficace, il doit y avoir une expérience contradictoire. La TE semble produire plusieurs expériences contradictoires possibles, notamment :

- Une diminution des affects et des émotions en raison des effets de la mémoire de travail, puisque des ressources exécutives centrales sont nécessaires pour accéder à/écarter un souvenir, avec des MO simultanés (Gunter \& Bodner, 2008 ; Shapiro, 2001)

- Des effets de détente en raison des effets physiologiques des MO (Kreyer \& Egon, 2008 ; Schubert et coll., 2011)

- Un sentiment d'efficacité en raison de la capacité à accéder à puis à écarter le souvenir de manière répétée

- La position de l'observateur et la posture de pleine conscience, apportant un sentiment de distance et d'acceptation

- Une inhibition des mécanismes de défense conscients, empêchant une dissociation potentielle, un évitement ou une abréaction

- Une consolidation de la perspective passéprésent en raison des déplacements de l'attention fréquents et brefs

Traitement adaptatif de l'information. En cohérence avec le modèle du traitement adaptatif de l'information (TAI) de la résolution EMDR du trauma, à mesure que le patient adopte de manière répétée une position qui est de plus en plus celle de l'observateur vis-à-vis du souvenir traumatique, l'attention se porte sur la perspective adulte adaptative du patient. En appliquant le modèle de Shapiro (2001), nous émettons l'hypothèse que la procédure TE pourrait établir de nouvelles voies neurales entre le réseau mnésique du trauma et les réseaux mnésiques associés à ces nouvelles perspectives, et que ceci pourrait favoriser la guérison et peut-être une certaine correction des distorsions cognitives.

\section{Différences entre la technique éclair et} d'autres formes de titrage

Contrairement à la plupart des autres formes de titrage, la TE aide le patient à se centrer sur le souvenir perturbant brut, mais pour une période très limitée. Le temps de contact avec le souvenir est ce qui est titré. Le laps de temps accordé dans la TE est beaucoup plus court que dans les autres formes de titrage. À la différence d'autres formes de titrage, la TE est extrêmement rapide. Aussi, avec la TE, les patients semblent presque déconnectés émotionnellement du souvenir et il est parfois difficile de déterminer à partir de la seule observation si un changement s'est produit.

\section{Limites de la technique éclair}

Bien que la TE paraisse réduire considérablement la perturbation, elle ne traite généralement pas complètement les souvenirs ni ne réduit leur perturbation à 0 . Le patient n'a pas le bénéfice d'une évaluation pour clarifier les composants de la cible. De plus, si des canaux multiples contribuent à la perturbation du patient, la TE, telle qu'elle est pratiquée actuellement, souvent n'attire pas l'attention sur des canaux secondaires ni n'a un impact sur eux. Dans le traitement TE, les cognitions ne sont pas abordées. Comme il n'y a pas de phase d'évaluation préalable, les distorsions cognitives ne sont pas identifiées au départ et souvent ne sont pas pleinement tirées au clair dans le traitement, même si certaines perspectives adultes émergent le plus souvent. Les patients qui sont très dissociatifs ou qui sont autrement incapables de se sentir raisonnablement calmes et en sécurité, avant même de se centrer sur le matériel perturbant, ont besoin d'aide pour se connecter à un état calme. S'ils n'y parviennent pas, ils ne sont pas de bons candidats à la TE. De plus, la TE a peu de chances d'être pleinement efficace si le souvenir cible est alimenté par un souvenir plus ancien qui n'est pas abordé.

\section{Aucune réponse indésirable signalée chez les patients}

À ce jour, les résultats d'environ 200 séances employant cette technique ont été recueillis de façon informelle. Aucune réponse indésirable n’a été signalée chez les patients, à part quelques abréactions qui ont aisément répondu à une série standard de SBL rapides avec des commentaires de cadence. 
Indications pour l'emploi de la technique éclair

Il faut souligner que la TE est recommandée pour la phase de préparation de l'EMDR ou pour résoudre un traitement bloqué dans la phase de désensibilisation. Elle n'est pas présentée comme le substitut d'une prise en charge EMDR complète. Cette technique semble la plus utile pour les patients qui ne sont pas fortement dissociatifs mais qui résistent à accéder pleinement à une perturbation intense. Bien qu'un clinicien EMDR hautement qualifié pourrait finir par persuader un tel patient à accéder pleinement à un souvenir très perturbant, la TE l'accomplit d'une manière relativement dépourvue d'effort et indolore, et les patients semblent devenir moins craintifs à l'idée de devenir submergés.

\section{Facilité d'apprentissage}

Les cliniciens EMDR semblent apprendre la technique assez facilement. Parmi la vingtaine de cliniciens formés à l'EMDR qui ont reçu au moins vingt minutes de formation et regardé trois courtes vidéos montrant la technique, tous ont rapporté avoir obtenu quelques résultats positifs avec la TE. Une formation plus détaillée améliore les résultats.

\section{Recommandations pour les recherches futures}

Une étude comparant l'efficacité auprès de deux groupes de patients appariés possédant des souvenirs extrêmement perturbants, l'un bénéficiant d'une prise en charge EMDR standard et l'autre de l'EMDR avec la TE dans la phase de préparation, aiderait à établir dans quelle mesure cette technique apporte une différence.

Une étude conçue pour déterminer la durée optimale des éclairs serait très utile. Il est possible que la préparation des patients à la TE en leur montrant une vidéo brève illustrant différentes durées des éclairs et en leur indiquant la durée optimale, réduirait davantage la courbe d'apprentissage pour les patients dont les éclairs sont trop longs au départ.

Autrement, l'enseignement de la TE en commençant par une cible moins perturbante, comme une scène perturbante dans un film, pourrait permettre aux patients d'apprendre à faire des éclairs avec une exposition minimale au souvenir. On peut supposer qu'une étude qui déterminerait si la TE devient plus facile et plus rapide pour les patients qui ont appris au départ comment faire des éclairs assez brefs sur leurs souvenirs et si cela accélère ou diminue l'efficacité de la TE, serait utile pour évaluer l'hypothèse de la mémoire de travail dans ses rapports avec la TE.
Questions supplémentaires. La TE nécessite-t-elle des $M O$ ? Il serait précieux de mener une recherche comparant la TE avec MO à la TE sans $\mathrm{MO} / \mathrm{SBL}$. Il serait également intéressant de comparer la TE avec MO à la TE avec tapotements bilatéraux et/ou des stimulations auditives. De plus, une recherche évaluant chacun des mécanismes d'action proposés dans cet article améliorerait la compréhension de la TE.

\section{Conclusion}

Cet article a décrit quatre prises en charge utilisant la TE, une méthode permettant de rapidement réduire les niveaux de perturbation en préparation du traitement EMDR de traumas. La technique semble efficace, rapide, bien tolérée et facilement enseignée aux praticiens. À ce jour, nous n'avons pas identifié de facteur de risque significatif associé à son utilisation. Des études supplémentaires sont justifiées.

\section{Bibliographie}

Barreiro, K. A., Suárez, L. D., Lynch, V. M., Molina, V. A., \& Delorenzi, A. (2013). Memory expression is independent of memory labilization/reconsolidation. Neurobiology of Learning and Memory, 106, 283-291.

Dudai, Y. (2004). The neurobiology of consolidations, or, how stable is the engram? Annual Review of Psychology, 55, 51-86.

Ecker, B., Ticic, R., \& Hulley, L. (2012). Unlocking the emotional brain: Eliminating symptoms at their roots using memory reconsolidation. New York, NY: Routledge.

Elsey, J. W., \& Kindt, M. (2017). Tackling maladaptive memories through reconsolidation: From neural to clinical science. Neurobiology of Learning and Memory, 142, 108-117.

Forgash, C. (2004, June). Healing the heart of trauma: Restoring connections and stability. Paper presented at the $5^{\text {th }}$ EMDR Europe Association Conference, Stockholm, Sweden.

Gomez, A. (2014, September). There and back again: The intricate and fascinating journey of helping children with complex trauma using EMDR therapy. Paper presented at the 20th EMDR International Association Conference, Denver, CO.

Greenwald, R. (2008). Progressive counting for trauma resolution: Three case studies. Traumatology, 14(4), 83-92.

Gunter, R. W., \& Bodner, G. E. (2008). How eye movements affect unpleasant memories: Support for a working- memory account. Behaviour Research and Therapy, 46, 913-931.

Hackmann, A., Ehlers, A., Speckens, A., \& Clark, D. M. (2004). Characteristics and content of intrusive memories in PTSD and their changes with treatment. Journal of Traumatic Stress, 17(3), 231-240. http://dx.doi. org/10.1023/b:jots.0000029266.88369.fd 
Hupbach, A., Gomez, R., Hardt, O., \& Nadel, L. (2007). Reconsolidation of episodic memories: A subtle reminder triggers integration of new information. Learning \& Memory, 14(1-2), 47-53.

Kinowski, K. (2003). Put your best foot forward: An EMDRrelated protocol for empowerment using somatosensory and visual priming of resource experiences. Retrieved from http: / / www.krystynakinowski.com/manual.html

Knipe, J. (2008, June). EMDR toolbox. Paper presented at the $9^{\text {th }}$ EMDR Europe Association Conference, London, United Kingdom.

Korn, D. L., \& Leeds, A. M. (2002). Preliminary evidence of efficacy for EMDR resource development and in- stallation in the stabilization phase of treatment of complex posttraumatic stress disorder. Journal of Clinical Psychology, 58(12), 1465-1487. http://dx.doi.org/10.1002/ jclp.10099

Kreyer, A. K., \& Egon, S. (2008, June). Physiological effects of eye movements of different speeds and eye fixation during engagement in negative autobiographical memories: Experimental research regarding EMDR. Paper presented at the $9^{\text {th }}$ EMDR Europe Association Conference, London, United Kingdom.

Lanius, R. A., Vermetten, E., Loewenstein, R. J., Brand, B., Schmahl, C., Bremner, J. D., \& Spiegel, D. (2010). Emotion modulation in PTSD: Clinical and neurobiologi- cal evidence for a dissociative subtype. The American Journal of Psychiatry, 167(6), 640-647. http: / / dx.doi. org/ 10.1176/appi.ajp.2009.09081168

Lanius, U. (2005, April). 'Dissociative processes' and EMDRstaying connected. Paper presented at $3^{\text {rd }}$ Annual Conference of the EMDR Association UK and Ireland, Jordanstown, Northern Ireland.

Lee, J. L. (2009). Reconsolidation: Maintaining memory relevance. Trends in Neurosciences, 32(8), 413-420.

Leutner, S., \& Cronauer, E. (2010). Stabilizing while processing -integration of resources into the EMDR protocol (RIT). Paper presented at the $11^{\text {th }}$ EMDR Europe Association Conference, Hamburg, Germany.

Levine, P. (1997). Waking the tiger-healing trauma. Berkeley, CA: North Atlantic Books.

Manfield, D. C. (1986). Computer-assisted weight-loss: A subliminal and behavioral methodology for motivated females (Unpublished doctoral dissertation). University of Wisconsin, Madison, WI.

Manfield, P. (2010). Dyadic resourcing: Creating a foundation for processing trauma. Berkeley, CA: Cornucopia.

Manfield, P. (2013). EMDR up close: Subtleties of trauma processing. Berkeley, CA: Cornucopia.

Manfield, P., \& Shapiro, F. (2003). The application of EMDR to the treatment of personality disorders. In J. F. Magnavita (Ed.), Handbook of personality disorders: Theory and practice (pp. 304-330). New York, NY: Wiley.
Mansfield, D. J. (1997). Subliminal priming and mood: A preliminary study (master's thesis). University of Natal, Peitermaritzburg, South Africa.

Maxfield, L., Melnyk, W. T., \& Hayman, G. C. (2008). A working memory explanation for the effects of eye movements in EMDR. Journal of EMDR Practice and Research, 2(4), 247-261. http://dx.doi. org/10.1891/1933-3196.2.4.247

Meyer, C., \& Waller, G. (1999). The impact of emotion upon eating behavior: The role of subliminal visual processing of threat cues. International Journal of Eating Disorders, 25(3), 319-326. http://dx.doi.org/10.1002/ (sici)1098-108x(199904)25:33.0.co;2-9

Ochberg, F. (1996). The counting method for ameliorating traumatic memories. Journal of Traumatic Stress, 9, 873-880.

Pedreira, M. E., Pérez-Cuesta, L. M., \& Maldonado, H. (2004). Mismatch between what is expected and what actually occurs triggers memory reconsolidation or extinction. Learning \& Memory, 11(5), 579-585.

Schubert, S. J., Lee, C. W., \& Drummond, P. D. (2011). The efficacy and psychophysiological correlates of dual- attention tasks in eye movement desensitization and reprocessing (EMDR). Journal of Anxiety Disorders, 25(1), $1-11$.

Sevenster, D., Beckers, T., \& Kindt, M. (2013). Prediction error governs pharmacologically induced amnesia for learned fear. Science, 339(6121), 830-833.

Shapiro, F. (1989). Efficacy of the eye movement desensitization procedure in the treatment of traumatic memories. Journal of Traumatic Stress, 2(2), 199-223.

Shapiro, F. (2001). Eye movement desensitization and reprocessing (EMDR): Basic principles, protocols, and procedures ( $2^{\text {nd }}$ ed.). New York, NY: Guilford Press.

Silverman, L. H., \& Weinberger, J. (1985). Mommy and I are one: Implications for psychotherapy. American Psychologist, 40(12), 1296-1308. http://dx.doi. org/10.1037/0003-066x.40.12.1296

Van Veen, S. C., Van Schie, K., Wijngaards-De Meij, L. D., Littel, M., Engelhard, I. M., \& Van den Hout, M. A. (2015). Speed matters: Relationship between speed of eye movements and modification of aversive autobiographical memories. Frontiers in Psychiatry, 6, 45. http: / / dx.doi.org/10.3389/fpsyt.2015.00045

Wolpe, J. (1958). Psychotherapy by reciprocal inhibition. Stanford, CA: Stanford University Press.

Merci d'adresser toute correspondance concernant cet article à Philip Manfield, $\mathrm{PhD}$, John F. Kennedy University, 2936 Domingo Ave., Suite 4, Berkeley, CA 94705, ÉtatsUnis. Courriel : philip.manfield@gmail.com 